\title{
Factors related to endocrine changes and hormone substitution treatment during pre- and post-operation stages in craniopharyngioma
}

\author{
FENGLEI SUN ${ }^{1}$, XINTANG SUN ${ }^{2}$, XIAOLONG DU ${ }^{1}$, HONGSHUN XING ${ }^{1}$ and BIN YANG ${ }^{3}$ \\ ${ }^{1}$ Department of Neurosurgery, Weifang People's Hospital, Weifang, Shandong 261000; ${ }^{2}$ Department of Food \\ and Pharmaceutical Science and Technology, Shandong Vocational Animal Science and Veterinary College, \\ Weifang, Shandong 261061; ${ }^{3}$ Department of Neurosurgery, Affiliated Hospital of Weifang Medical University, \\ Weifang, Shandong 261031, P.R. China
}

Received March 3, 2016; Accepted November 2, 2016

DOI: $10.3892 / \mathrm{ol} .2016 .5418$

\begin{abstract}
Factors related to endocrine changes and hormone substitution treatment during pre- and post-operation periods in craniopharyngioma cases were examined. Twenty patients who underwent tumor resection from January 2012 to January 2015 were included in the study. We monitored factors related to endocrine changes and hormone substitution treatment during pre- and post-operation periods. Blood thyroid-stimulating hormone, triiodothyronine, thyroxine, prolactin, follicle-stimulating hormone, luteinizing hormone and cortisol levels were measured in the patients. Urinary volume and urine specific gravity were also measured before and after the operation. After the operation, we observed diabetes insipidus and a decrease in TSH and gonadal hormone levels more frequently compared to pre-operation. However, incidence of high prolactin was markedly reduced following surgery. The number of hypothyroidism cases before and after surgery had no statistical significance. In conclusion, surgery for craniopharyngioma aggravated endocrine dysfunction. Craniotomy and total tumor resection had considerable effects on the endocrine system. Routine hormone therapy as an alternative treatment was necessary after operation. Alternative treatment of adrenal cortex hormones reduced the incidence on low blood cortisol.
\end{abstract}

\section{Introduction}

Craniopharyngioma is a common benign tumor in sellar area and mainly originates from pituitary and pituitary stalk, accounting for up to $3 \%$ of all intracranial tumors (1).

Correspondence to: Dr Bin Yang, Department of Neurosurgery, Affiliated Hospital of Weifang Medical University, 2428 Yuhe Road, Weifang, Shandong 261031, P.R. China

E-mail: yang_bin1212@163.com

Key words: craniopharyngioma, drug substitution therapy, endocrine, resection
As craniopharyngioma mainly grows in midline of suprasellar-hypothalamus and maintains a close relationship with adjacent nerves, pituitarium, hypothalamus and blood vessels, despite its appearance of a benign tumor, it is likely to cause postoperative associated complications if microsurgeries are performed (2). Most patients with hypothalamus-pituitary disorder are likely to have endocrine dysfunction (3). Patients' postoperative hormone conditions, life quality, growth and development are gradually observed by neurosurgeons, and their hormone conditions in the perioperative period may influence the prognosis of the patients.

The present study retrospectively examined 20 patients with craniopharyngioma who underwent neurosurgical procedures from January 2012 to January 2015. Factors that influence patients' endocrine changes in the perioperative period and the application of hormone replacement were analyzed to determine the prognosis and any complications arising.

\section{Patients and methods}

Clinical data and clinical manifestation. Twenty patients, 12 men and 8 women, aged 15-65 years (average 42.5 years) were included in this study. The course of disease for these cases ranged from 1 to 36 months (5 months on average). Of the 20 patients, 18 patients had headaches and 10 suffered from visual disorders. Two patients consumed alcohol frequently and had polyuria while 8 suffered from nausea and vomiting. Five patients had hypoevolutism, 4 patients experienced memory loss, while 1 patient had amenorrhea.

Magnetic resonance imaging (MRI) and computed tomography $(C T)$ scanning. All patients had MRI and CT scan before operation and results showed 3 tumors in supra seller and hypothalamus, 2 in the third ventricle, 2 in intrasellar-supraseller and 3 from upslope to brain stem. In total, 13 tumors were cystic with solid structure, 2 were solid tumors and 4 had cystic structure.

Surgical methods and pathological situation. All 20 patients underwent frontal basal interhemispheric approach, coronal 
Table I. Relationship between changes in endocrine hormone levels and the size of tumor resection.

\begin{tabular}{|c|c|c|c|c|c|c|}
\hline \multirow[b]{2}{*}{ Endocrine changes } & \multicolumn{2}{|c|}{ Total resection $(\mathrm{n}=18)$} & \multicolumn{2}{|c|}{ Sub-resection $(n=12)$} & \multicolumn{2}{|c|}{ Total $(n=20)$} \\
\hline & Before & After & Before & After & Before & After \\
\hline Hypothyroidism & 2 & 2 & 1 & 1 & 3 & 3 \\
\hline TSH insufficiency & 3 & 8 & 1 & 1 & 4 & 9 \\
\hline Blood cortisol & 5 & 0 & 1 & 0 & 6 & 0 \\
\hline Sex hormone insufficiency & 6 & 9 & 1 & 1 & 7 & 10 \\
\hline HPPL & 5 & 1 & 1 & 0 & 6 & 1 \\
\hline DI & 6 & 11 & 1 & 1 & 7 & 12 \\
\hline
\end{tabular}

After alternative treatment with cortisol hormone, there was no adrenal cortical insufficiency by cortisol one week later. DI condition in 1 patient relieved after operation. TSH, thyroid-stimulating hormone; DI, diabetes insipidus; HPPL, human phospholipases.

incision in bilateral frontal hairline with single frontal craniotomy. After operation and before discharging from hospital, all patients were re-examined with MRI and CT scan. Results of re-examination revealed that in 18 patients tumors were totally removed, while in 2 cases tumors were still detectable after operation and clinical pathological tests revealed craniopharyngioma.

Endocrine hormone test during pre-operation period. Morning fasting blood tests were performed on all patients in order to measure thyroid-stimulating hormone (TSH), triiodothyronine, thyroxine, prolactin (PRL), follicle-stimulating hormone and luteinizing hormone levels. Blood cortisol level was also measured twice per day at 8:00 a.m. and 4:00 p.m. Urinary volume and specific gravity was also tested prior to operation. One week after operation we repeated these tests.

Hormone use during pre-operation period. Before operation, patients who suffered from adrenal cortical insufficiency received a daily dose of 100-200 mg of hydrocortisone orally. Patients with no adrenal cortical insufficiency received 15-20 mg/day. All patients received $100 \mathrm{mg}$ hydrocortisone during the operation. Three days after operation, all patients received a daily dose of 200-300 $\mathrm{mg}$ of hydrocortisone intravenously. Three days later, patients were administered $15-30 \mathrm{mg} /$ day prednisone orally. According to different conditions such as blood and urinary-free-cortisol levels, we changed the dosage. For patients with hypothyroidism we prescribed $50 \mathrm{mg} /$ day of levothroid at the beginning and increased the dose up to $100-200 \mathrm{mg} /$ day during a few weeks.

Statistical analysis. All data were processed using software SPSS 13.0 (SPSS, Inc., Chicago, IL, USA). In Chi-square test, $\mathrm{P}<0.05$ was considered to indicate a statistically significant difference.

\section{Results}

Patients. Before the operation, 18 cases suffered from at least 1 hormone insufficiency (PRL excluded). They were all in poor condition after the operation which agreed with the prior reports showing that there was no clear recovery early after operation.
Thyroid function. We did not have any new cases of hypothyroidism after the operation. After operation the incidence of TSH insufficiency was increased significantly $(\mathrm{P}<0.05)$, similarly to total tumor resection. There was no statistical significance to those who underwent total tumor sub-resection.

Adrenal cortical function, sex hormone and PRL. The patients received cortisol replacement treatment and none of them experienced any reduction in cortisol level when re-examined one week after the operation. The incidence of sex hormone insufficiency after operation was higher than before for patients who received total tumor resection $(\mathrm{P}<0.05)$. While there was no significant change regarding the incidence of sex hormone insufficiency for those who underwent total tumor sub-resection.

In the case of hyperprolactimia, we saw significant improvement before and after operation $(\mathrm{P}<0.05)$. Additionally, the condition of the patients who underwent total tumor resection was improved significantly $(\mathrm{P}<0.05)$.

Diabetes insipidus (DI). The incidence of DI was significantly higher after operation. Among all the patients, one with DI before operation experienced relief after operation.

There was statistical significance to total tumor resection before and after operation in total resection cases, but no significant difference was observed in sub-resection cases.

For those cases with TSH insufficiency and sex hormone insufficiency before operation, we had no cured cases after operation. Additionally, PRL levels did not show any increase in those cases who had normal level of PRL before the operation. We found no link between endocrine hormone level changes and the size of the resected tumor (Table I).

\section{Discussion}

Hypothyroidism is a common endocrine symptom of craniopharyngioma. However, owing to long cycle period of thyroid hormone, there is no strong effect at the early time. By contrast, TSH is affected more markedly $(4,5)$. After surgery, the incidence of low cortisol may go up to $24-91 \%$ (6).

The report mentioned that after a craniotomy the rate was $61 \%$ (6). All the patients received adrenocortical hormone as an alternative treatment, thus no one had hormone 
insufficiency one week after operation. We found that alternative cortisol hormone treatment was the optimal choice to reduce the incidence of low cortisol during pre-operation period. Low sex hormone, particularly in adult men, is a common symptom of craniopharyngioma which is usually overlooked. Changes in sex hormone levels can cause serious damage to patients in severe conditions. In these patients PRL may be increased by $8-50 \%$, which is a sign of hypothalamus invasion or impediment in dopamine transmission between hypothalamus and hypophyseal portal system (7).

Our results showed that PRL levels were reduced significantly after operation. However, in 2 patients the PRL level did not return to normal. One of them underwent a tumor sub-resection while the other had a total tumor resection using transphenoidal approach. More evidence is needed to establish whether PRL can be used as an evaluation indicator to predict the probability of recurrence. On the other hand, it has been shown that surgery would not impact the high incidence of PRL (8). DI is one of clinical features to craniopharyngioma and tumor has an impact on antidiuretic hormone creation and transmission which can lead to large volumes of hypostheuria. This condition is a result of poor water resorption from distal convoluted renal tubules and collecting tubules. Operation can have an impact on DI due to the close relation between craniopharyngioma and pituitary stalk, thus any kind of damage, even small, to pituitary may cause severe DI (8). Our results demonstrated that DI condition worsen after operation except in one case that DI was relieved after operation. We speculated that the relieved DI was due to a recovery in creation and transmission system. Our results showed that total tumor resection negatively affected the endocrine system and increased the incident of endocrine disorder and worsen the DI condition. Nevertheless, we should pay attention to this one DI relief case who had a total tumor resection, which suggested that having less load on hypothalamus and pituitary might be good for DI.

The probability of successful treatment in cases of endocrine dysfunction caused by craniopharyngioma is very poor (9). In most cases, the condition can degenerate after surgery. Problems associated with adrenal cortical, thyroid and gonadal functions may have a great impact on patient recovery and hormone replacement therapy is a crucial step in craniopharyngioma treatment (9). Thus, overall evaluation of endocrine system should be made prior to surgery.

In this study, regular patients received $15 \mathrm{mg}$ of prednisone orally 3 days before operation while patients with adrenal cortical dysfunction had a daily dose of 100-200 mg of hydrocortisone. During operation, patients received $100 \mathrm{mg}$ of hydrocortisone and subsequently 3 days after operation, an oral dose of 15-20 mg of hydrocortisone was given (this dose was reduced gradually). During the whole process, blood cortisol level as well as urinary free-cortisol was measured. When clinical symptoms such as hyperpyrexia, infection and trauma occurred suddenly, we increased the dose of hydrocortisone (intravenous injection of 100-300 mg/day) until the symptoms disappeared. Few days later, we decreased the dose to the level used before the increase. In some cases patients must continue taking hydrocortisone for the rest of their lives. In the case of hypothyroidism, patients took levothroid after operation (50 $\mu \mathrm{g}$ at the beginning which was gradually increased to 100-200 $\mu \mathrm{g}$ during following weeks). Symptoms such as fear of cold, poor mental condition and edema were relieved under this treatment. Adrenal cortical function might degenerate if patients receive thyroid hormone alone, however, patients who received a combination of thyroid hormone with cortisone hormones were generally in better condition. Men with sex hormone insufficiency received testosterone which helped them in their protein synthesis, physical strength and state of nutrition. Women received a small amount of male hormone to improve their gender function and physical strength.

Total tumor resection is the first choice and the most important factor to reduce occurrence the complication. We should protect hypothalamus, pituitary stalk and pituitary against unnecessary stretch or resection. Furthermore, endocrine alternative treatment should be used.

\section{References}

1. Yamini B and Narayanan M: Craniopharyngiomas: An update. Expert Rev Anticancer Ther 6: S85-S92, 2006.

2. Bahuleyan B, Menon G and Nair S: Immediate postoperative death due to hypothalamic injury following surgery for craniopharyngioma. J Clin Neurosci 16: 850-851, 2009.

3. Puget S, Garnett M, Wray A, Grill J, Habrand JL, Bodaert N, Zerah M, Bezerra M, Renier D, Pierre-Kahn A and SainteRose C: Pediatric craniopharyngiomas: classification and treatment according to the degree of hypothalamic involvement. J Neurosurg 106 (Suppl 1): 3-12, 2007.

4. Campbell PG, McGettigan B, Luginbuhl A, Yadla S, Rosen M and Evans JJ: Endocrinological and ophthalmological consequences of an initial endonasal endoscopic approach for resection of craniopharyngiomas. Neurosurg Focus 28: E8, 2010.

5. Kassam AB, Gardner PA, Snyderman CH, Carrau RL, Mintz AH and Prevedello DM: Expanded endonasal approach, a fully endoscopic transnasal approach for the resection of midline suprasellar craniopharyngiomas: A new classification based on the infundibulum. J Neurosurg 108: 715-728, 2008.

6. Kunihiro N, Goto T, Ishibashi K and Ohata K: Surgical outcomes of the minimum anterior and posterior combined transpetrosal approach for resection of retrochiasmatic craniopharyngiomas with complicated conditions. J Neurosurg 120: 1-11, 2014.

7. Hori T, Kawamata T, Amano K, Aihara Y, Ono M and Miki N: Anterior interhemispheric approach for 100 tumors in and around the anterior third ventricle. Neurosurgery 66 (Suppl Operative 3): 65-74, 2010.

8. Jung TY, Jung S, Choi JE, Moon KS, Kim IY and Kang SS: Adult craniopharyngiomas: Surgical results with a special focus on endocrinological outcomes and recurrence according to pituitary stalk preservation. J Neurosurg 111: 572-577, 2009.

9. Garrè ML and Cama A: Craniopharyngioma: Modern concepts in pathogenesis and treatment. Curr Opin Pediatr 19: 471-479, 2007. 